\title{
Analysis and Development Enlightenment on Source Term Inversion Technology of Nuclear and Chemical Hazards
}

\author{
Han Chaoshuai ${ }^{1,2}$, Zhu Xuezheng ${ }^{*}, 1$, Gu Jin ${ }^{1}$, Jiang Jinli ${ }^{1}$, Ma Yan ${ }^{1}$, Zhou Qiang ${ }^{1}$, Chen Chen ${ }^{1}$ \\ ${ }^{1 .}$ Institute of NBC Defense, Beijing, 100000; \\ 2. State Key Laboratory of NBC Protection for Civilian, Beijing, 102205
}

\begin{abstract}
Source Term Inversion (STI) is of great significance to mitigate and contain the sources of nuclear and chemical hazards, accurately predict the spatiotemporal transmission and diffusion of nuclear and chemical hazards, assist combat operations and support decision-making. This paper summarizes and analyzes the key algorithms and application platform of the current nuclear and chemical hazard source inversion technology, and puts forward the enlightenment and suggestions for its development, which has a certain theoretical reference and reference value.
\end{abstract}

\section{Introduction}

Usually, nuclear and chemical attacks are accompanied by sudden and huge lethality. It is an important task to determine the information related to the nuclear, chemical and biological safety and national defense security of the country, which can help to alleviate and contain pollution sources, and more accurately predict the spatiotemporal transmission and diffusion of pollutants, providing a decision for the army's combat protection, combat decontamination and combat operations Policy basis ${ }^{[1]}$. Therefore, based on the inversion technology of nuclear and chemical hazards source term, this paper summarizes the relevant methods, technologies and platforms, analyzes the focus of next research.

\section{Key Algorithm of Nuclear and Chemical Hazards STI}

\subsection{STI based on Euler method}

Most source term inversion algorithms use Euler method to obtain source term information by minimizing the difference function, such as optimal interpolation method, genetic algorithm, Kalman filter, ensemble Kalman filter, four-dimensional variation, set four-dimensional variation, etc. ${ }^{[2-6]}$. Euler methods usually either rely on statistical methods for selecting test solutions or use adjoint models to calculate backward from observation time to release time. Both methods may include iterative optimization solution ${ }^{[7-9]}$.

In Bayes formula and Kalman filter, random sampling is used to generate source information, which is then used as the input of AT\&D model ${ }^{[10-12]}$. The difference between the subsequent concentration output and the concentration observation can be used to determine the possibility of these initial estimates. All probability density functions of source information generated by all estimation likelihood calculations can be used to optimize the program to obtain iterative improved estimates of source locations ${ }^{[13-16]}$. In addition, Bourne Joseph R et al. [17], of the Automatic Machine Control Laboratory, Department of Mechanical Engineering, University of Utah, USA, proposed a new algorithm for plume source term estimation and source search motion planning based on nonparametric Bayes, which uses the coordination and plume estimation model among multiple robots to realize faster and more robust pollution source location determination and source intensity estimation. The algorithm has been used in the design of mobile robots equipped with gas concentration sensors.

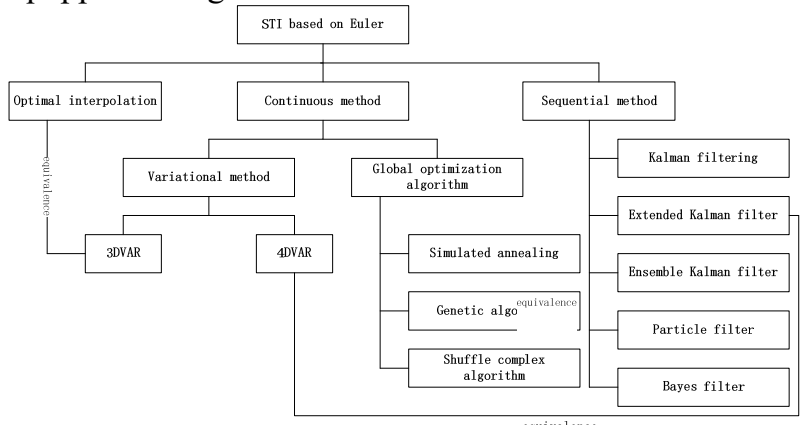

Fig.1 source term inversion algorithm based on Euler method

Many Euler STI contain adjoint models. On the basis of adjoint model, variational assimilation method is gradually applied to source term inversion, and a large number of experiments and applications have been carried out in the research process. The most representative is the CEREA research team headed by Marc Bocquet ${ }^{[18]}$, which has successively verified the effectiveness of variational

hanzaoshuai@163.com. 
data assimilation in the inversion of nuclear accident source terms by using numerical simulation experiments, wind tunnel experimental data and European atmospheric diffusion experimental data, and then estimated and studied the release source terms of Chernobyl and Fukushima nuclear accidents.

In addition, there are other Euler methods that do not require adjoint model or Bayesian reasoning. By iteratively adjusting the AT\&D prediction driven by source information, these methods directly obtain the source information from the decentralized model to match the observed pollution concentration values.

\subsection{STI based on Lagrange method}

The second type of STI algorithm is Lagrange method, but it is not widely studied and applied as Euler algorithm. Lagrange methods belong to the category of entity backtracking, that is, they trace the state of an entity to its original state. This method is similar to the target tracking problem proposed by Hall and McMullen ${ }^{[19]}$ (2004). The traditional Lagrangian backtracking method is to trace the source by analyzing the time of a single fluid package ${ }^{[20-}$ 22]. The requirements of wind field and pollutant concentration data are often too high to be used for source term estimation. In addition, the reverse flow must converge in order to accurately trace the fluid back to its source, or the fluid must evolve in time so that multiple trajectories intersect at the source ${ }^{[23]}$.

Based on the traditional Lagrangian package backtracking method, some scholars have extended and improved it from the perspective of multi-source inversion and multi-scale inversion, and proposed Lagrange entity backtracking, Lagrange particle model and other methods. Andrew J. Annunzio, Sue Ellen Haupt and others [24-27] (2007, 2012) , of Pennsylvania State University, have carried out a series of studies in the field of pollution transmission and diffusion. In view of the complex problem of the number of uncertain pollution sources and the overlapping of emission, they proposed a multi entity field approximation (MEFA) method based on Lagrange state estimation to locate the location of multiple instantaneous or continuous release pollution sources by integrating the effects of turbulence and solid diffusion on multi-source concentration field. However, the influence of complex underlying surfaces such as buildings and terrain on pollutant diffusion is not considered in the literature.

Stohl A of Greece used the Lagrange particle model ${ }^{\text {[28] }}$ in RODOS to conduct STI research based on data of numerical simulation experiment and Mol Belgium site tracer experiment, which preliminarily realized the inversion of the release source term of nuclear accident using dose rate data. Subsequently, in order to strengthen the ability of RODOS to predict and evaluate the consequences of nuclear accidents, they launched the project of "data assimilation of off-site nuclear accident emergency" based on Kalman filter ${ }^{[29]}$. Unfortunately, due to the complexity of the actual nuclear accident, the experimental results are quite different from the actual situation, so it has not been carried out all the time ${ }^{[30]}$.
Mahmoud Bady ${ }^{[31]}$ (2017) carried out Lagrangian particle inversion modeling according to the basic principles of computational fluid dynamics, aiming to determine the location of urban air pollution sources by using direct inversion technology.

\subsection{STI based on deep learning}

Deep learning provides a new idea for source term inversion. It does not need to explore the mathematical relationship between the source term and the observed value, but only needs to learn enough training data to quickly predict and retrieve. The mapping relationship of deep learning organization is shown in Figure 2. Ilias Bougoudis et al. ${ }^{[32]}$ (2015) proposed a triple intelligent integrated system (HISYCOL) based on hybrid machine learning. Through cluster data sets and unsupervised machine learning, the system realizes clustering tracking of data vectors and hidden knowledge mining, and has significant effect in processing correlation analysis and source term inversion under high pollutant concentration.

Jan Kleine, University of Twente, the Netherlands Deters et al. ${ }^{\text {[3] }}$ (2017) proposed a machine learning inversion method based on six-year meteorological data and PM2.5 pollution data to solve the problem of urban particulate matter pollution diffusion. The test and analysis show that the inversion accuracy of this method is better than that of stable weather under strong wind or high precipitation, because under stable weather, the influence of underlying surface on PM2.5 pollution diffusion is far greater than that under extreme environment.

Julien Brajard et al. ${ }^{[34]}$ (2020) of Sorbonne University in France proposed a hybrid data assimilation method based on the combination of Kalman filter and neural network to solve the problems of large noise impact in observation data and low accuracy of pollution prediction model, and carried out numerical experimental analysis through Lorenz 96 model. Compared with Kalman filter, the hybrid method can not only shorten the calculation time by two times, but also ensure the accuracy of inversion and prediction decline steadily with the increase of observation noise.

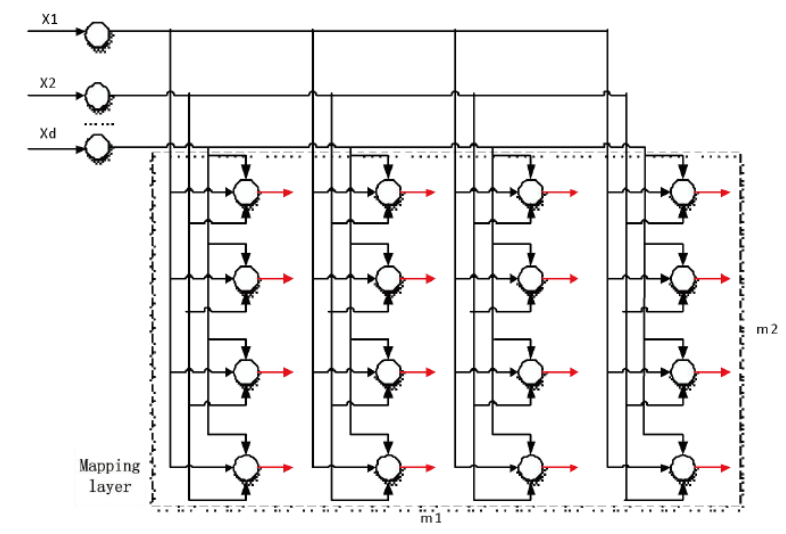

Fig.2 Example of deep learning organization mapping relationship 


\section{Application Platform of Nuclear and Chemical Hazards STI}

In Europe and the United States, the inversion of nuclear and chemical hazard source terms developed to the peak in the 1970s and 1980s, and the technical research system was basically formed ${ }^{[35]}$. Since the 1990s, with the development of relevant basic disciplines, inversion technology has been integrated with numerical analysis of micro meteorological field, pollutant diffusion of complex terrain, virtual environment simulation, etc. many countries, such as the United States, the United Kingdom, the Netherlands, Sweden and other countries have successively established comprehensive emergency response systems including leakage source model, wind field model and diffusion model, and have a variety of evaluation software systems, which have been widely used in the international business, and have reached a mature degree of industrialization. The most representative systems are $\mathrm{HG}, \mathrm{NARAC}$ and SAFER system.

HG system ${ }^{[36]}$ mainly includes thermodynamic model, escape model, plume jet model, heavy gas diffusion model, long-distance diffusion model and so on, which can evaluate the diffusion of gas and liquid or the two-phase release of multi-component mixture. It is used for gas escape, flash evaporation, evaporation tank, heavy gas diffusion, pure diffusion and UF6 gas diffusion with chemical reaction. NARAC system ${ }^{[37]}$ can simulate complex flow field, detailed particle diffusion, dry and wet deposition process on various spatial scales, including local and regional meteorological prediction, diffusion model and nuclear explosion settlement model, and can simulate and analyze the leakage and release of nuclear and chemical hazards in complex environment. SAFER system ${ }^{[38]}$ can handle various types of releases, including instantaneous, continuous, transient flows, ground level, uplift releases, and low or high momentum jets.

In addition, the British NAME system ${ }^{[39]}$ can retrieve and predict the instantaneous or continuous time air concentration, including the concentration, deposition and dose of radioisotopes, by tracking the three-dimensional trajectory of fluid particles and calculating the air concentration by Monte Carlo method, simulating the medium and long-term transport and deposition of pollutants. GASMAL ${ }^{[40]}$, a decision support system developed by the Netherlands Institute of Applied Sciences (TNO), combines calculation speed, graphic display and database information to enhance the fast decision-making, which is crucial for chemical accident emergency, and ensures the timeliness of emergency response. 
Table1. Nuclear and chemical hazards emergency response system

\begin{tabular}{ccccc}
\hline $\begin{array}{c}\text { Name } \\
\text { (Development Agency) }\end{array}$ & Mesoscale atmospheric diffusion model & $\begin{array}{c}\text { Wind field } \\
\text { calculation }\end{array}$ & $\begin{array}{c}\text { Hazardous } \\
\text { dose }\end{array}$ & $\begin{array}{c}\text { Mesoscale } \\
\text { and long } \\
\text { range models }\end{array}$ \\
\hline NARAC(LLNL/USA) & $\begin{array}{c}\text { Particle diffusion model } \\
\text { Gaussian plume model; } \\
\text { Gauss plume model; }\end{array}$ & Yes & Yes & Yes \\
Lagrange puff model & Yes & Yes & Yes \\
RESEY(FZK/ Germany) & Segmented Gaussian plume model & Yes & Yes & - \\
STREAM(SSES/USA) & Lagrange puff model (MESOI); & Yes & Yes & - \\
LENA-WIN(SSI/ Sweden) & Gaussian linear plume model & Yes & Yes & - \\
RECASS(SPA/ Russia) & Gaussian puff model; three dimensional & Yes & Yes & - \\
SPEEDI(JAERI/ Japan) & Particle diffusion mode (WIND04/PRWDA) & Yes & Yes & Yes \\
IMIS(BFS / Germany) & Lagrange and Euler diffusion models & Yes & Yes & - \\
WISERD(NRPB / Britain) & Gaussian class pattern & Yes & Yes & - \\
CONRAD(IRSN/ France) & Graph method and Gauss puff model & Yes & Yes & - \\
\hline
\end{tabular}

\section{Enlightenment to my development}

To sum up, the research on the inversion of source terms of nuclear and chemical hazards in foreign countries has been quite large. From the perspective of application platform, some countries such as the United States, the United Kingdom, the Netherlands and other countries have developed relatively mature inversion and diffusion prediction platforms; from the perspective of inversion algorithm, whether it is Euler principle, Lagrange principle or deep learning, each method gradually presents the trend of integration and integration.

\subsection{Deep Learning Will Become a New Research Hotspot}

Compared with the optimal interpolation method, genetic algorithm, Kalman filter and other methods, deep learning has obvious advantages in improving the accuracy and speed of hazard inversion, which brings new research ideas for source term inversion. However, deep learning requires high training samples and computational performance, and it is difficult to learn complex inversion samples, which is still in the primary research stage. The inversion algorithm based on deep learning, fourdimensional variation and ensemble Kalman filter is bound to become an important means to solve the inversion problem in complex environment.

\subsection{Inversion of Multi Pollution Sources Will Become a New Research Focus}

According to the research status, domestic and foreign scholars mainly focus on single point source continuous or instantaneous release inversion, and there are few studies on multi pollution sources term inversion. Some research institutions in the United States have carried out experimental research, and the domestic is still in the stage of mathematical derivation. However, in the actual combat environment, there is no single point source pollution. The inversion research of continuous, instantaneous and even mixed release of multiple pollution sources is more important for hazard prediction and protection decisionmaking. Therefore, the source term inversion of multiple pollution sources will be the focus of practical application in the next step.

\subsection{Qualitative and Quantitative Mixed Inversion Will Become a Research Difficulty}

At present, the information obtained by all kinds of reconnaissance and monitoring equipment in the battlefield environment is diverse and in different formats. Most of the monitored data are qualitative data based on sensitivity alarm, and the collection and acquisition methods of quantitative monitoring value data are relatively less. At the same time, there are many uncertainties in the acquisition of information such as pollution species, hazard source intensity, attack mode, meteorological field and so on, which is difficult to guarantee the error of the result. Therefore, it is a difficult problem to put forward the qualitative and quantitative hybrid inversion technology suitable for battlefield environment.

In addition, the scientific layout of monitoring equipment is of great significance to reduce redundant interference and improve inversion accuracy, but there are relatively few studies that can be used for reference, which is also a key link that needs to be strengthened.

\section{About the Author}

Han Chaoshuai, research assistant, born in 1993, Ph.D. student, research on chemical hazards source term inversion.

Zhu Xuezheng, professor, born in 1962, doctoral supervisor, research on NBC defence, email: hanzaoshuai@163.com.

\section{Reference}

1. Winiarek $\mathrm{V}$, Bocquet $\mathrm{M}$, Duhanyan $\mathrm{N}$, et al. 
Estimation of the caesium-137 source term from the Fukushima Daiichi nuclear power plant using a consistent joint assimilation of air concentration and deposition observations[J]. Atmospheric environment,2014, 82:268-279.

2. Camelli, F., Lohner, R.. Assessing maximum possible damage for contaminant release $[\mathrm{J}]$. Engineering Computations, 2004, 21: 748-760.

3. Chang, M.E., Hartley, D.E., Cardelino, C., HaasLaursen, D., Chang, W. L.. On using inverse methods for resolving emissions with large spatial inhomogeneities[J]. Journal of Geophysical Research , 1997,102: 16023-16036.

4. Haas-Laursen, D.E., Hartley, D.E., Prinn, R.G.. Optimizing an inverse method to deduce time-varying emissions of trace gases[J]. Journal of Geophysical Research,1996, 101:22823-22831.

5. Haupt, S.E.. A demonstration of coupled receptor/dispersion modeling with a genetic algorithm[J]. Atmospheric Environment,2005, 39: 7181-7189.

6. Thomson, L.C., Hirst, B., Gibson, G., Gillespie, S., Jonathan, P., Skeldon, K.D.,Padgett, M.J.. An improved algorithm for locating a gas source using inverse methods[J]. Atmospheric Environment, 2007, 41:1128-1134.

7. Bergin, M.S., Milford, J.B.. Application of Bayesian Monte Carlo analysis toa Lagrangian photochemical air quality model[J]. Atmospheric Environment, 2000,34:781-792.

8. Uliasz, M.. Application of the Perturbation theory to the Sensitivity analysis of an air Pollution model[J]. Zeitschrift fur Meterologie, 1983, 33: 355-362.

9. Uliasz, M., Pielke, R.A.. Application of the receptor oriented approach in mesoscale dispersion modeling[C]. In: van Dop, H., Steyn, D.G. (Eds.), Air Pollution Modeling and Its Application VIII. Plenum Press, New York, 1991: 399-407.

10. Haupt S.E.,Young G.S..Paradigms for source characterization[C].15th Joint Conference on the Applications of Air Pollution Meteorology with the A\&WMA, 88th Annual American Meteorological Society Conference, New Orleans, LA, 2008,January 21-25.

11. Rao, K.S.. Source estimation methods for atmospheric dispersion[J]. Atmospheric Environment, 2007, 41: 6963-6973.

12. Devaud C., Bushe W.K., Bellan J.. The modeling of the turbulent reaction rate under high-pressure conditions: A priori evaluation of the Conditional Source-term Estimation concept[J]. Combustion and Flame 2019 Vol.207:205-221.

13. Chow, F.K., Kosovic, B., Chan, S.. Source inversion for contaminant plume dispersion in urban environments using building-resolving simulations $[\mathrm{J}]$. Journal of Applied Meteorology and Climatology, 2007,47, 1553-1572.

14. Keats, A., Yee, E., Lien, F.-S. Bayesian inference for source determination with applications to a complex urban environment[J]. Atmospheric Environment, 2007a., 41:465-479.

15. Robins, P., Rapley, V.P., Green, N.. Real-time sequential inference of static parameters with expensive likelihood calculations[J]. Journal of the Royal Statistical Society, 2009, 58: 641-662.

16. Bourne, Joseph R., Pardyjak, Eric R., Leang, Kam K..Coordinated Bayesian-Based Bioinspired Plume Source Term Estimation and Source Seeking for Mobile Robots[J]. IEEE Transactions on Robotics 2019 Vol.35 No.4 P967-986.

17. Hourdin, F., Talagrand, O.. Eulerian backtracking of atmospheric tracers. I: adjoint derivation and parameterization of subgrid-scale transport[J]. Quarterly Journal of the Royal Meteorological Society, 2006, 132:567-583.

18. Marc Bocquet,Alban Farchi.On the consistency of the local ensemble square root Kalman filter perturbation update[J]. Tellus A: Dynamic Meteorology and Oceanography,2019,71(1):156-164.

19. Melina-Maria Zempila,Ronald J. van der A,Alkiviadis Bais, et al.TEMIS UV product validation using NILU-UV ground-based measurements in Thessaloniki, Greece[J].Atmospheric Chemistry and Physics,2017,17(11 Pt.2):7157-7174.

20. Melina Maria Zempila,Michael Taylor,Maria Elissavet Koukouli, et al.NILU-UV multi-filter radiometer total ozone columns: Comparison with satellite observations over Thessaloniki, Greece[J].Science of the Total Environment,2017, 590/591: 92-106.

21. Occhipinti, C., Aneja, V.P., Showers, W., Niyogi, D.. Back-trajectory analysis and source-receptor relationships: particulate matter and nitrogen isotopic compositionin rainwater[J]. Journal of Air andWaste Management Association, 2008,58:1215-1222.

22. Stohl, A.. Trajectory statistics-a new method to establish source-receptor relationships of air pollutants and its application to the transport of particulate sulfate in Europe[J]. Atmospheric Environment, 1996, 30:579-587.

23. Young, G.S., Limbacher J.A., Haupt S.E., Annunzio A.J.. Back Trajectories for Hazard Origin Estimation[C]. Seventh Conference on Artificial Intelligence and Its Applications to the Environmental Sciences at AMS Annual Meeting, Phoenix, AZ, 2009, January 11-15.

24. Allen, C.T., Haupt, S.E., Young, G.S.. Source characterization with a receptor/dispersion model coupled with a genetic algorithm[J]. Journal of Applied Meteorology and Climatology, 2007, 46:273287.

25. Andrew J. Annunzio, George S. Young, Sue Ellen Haupt..Utilizing state estimation to determine the source location for a contaminant[J]. Atmospheric Environment,2012,46:580-589. 
26. Andrew J. Annunzio, George S. Young, Sue Ellen Haupt..A Multi-Entity Field Approximation to determine the source location of multiple atmospheric contaminant releases[J]. Atmospheric Environment,2012,62: 593-604.

27. Luna Marie Rodriguez. Uncertainty propagation within source term estimation[D]. The Pennsylvania State University,2012.

28. Stohl A, Seibert P, Arduini J, et al. An analytical inversion method for determining regional and global emissions of greenhouse gases: Sensitivity studies and application to halocarbons[J]. Atmospheric Chemistry and Physics, 2009, 9(5): 1597-1620.

29. Stohl A, Prata A J, Eckhardt S, et al. Determination of time-and height-resolved volcanic ash emissions and their use for quantitative ash dispersion modeling: the 2010 Eyjafjallajokull eruption[J]. Atmospheric Chemistry and Physics, 2011, 11(9): 4333-4351.

30. Stohl A, Seibert P, Wotawa G, et al. Xenon-133 and caesium-137 releases into the atmosphere from the Fukushima Dai-ichi nuclear power plant: determination of the source term, atmospheric dispersion, and deposition[J]. Atmospheric Chemistry and Physics, 2012,12(5):2313-2343.

31. Mahmoud Bady. Evaluation of Gaussian Plume Model against CFD Simulations through the Estimation of CO and NO Concentrations in an Urban Area[J].American Journal of Environmental Sciences,2017,13(2):93-102.

32. Ilias Bougoudis, Konstantinos Demertzis, Lazaros Iliadis. HISYCOL a hybrid computational intelligence system for combined machine learning: the case of air pollution modeling in Athens[C]. Neural Comput \& Applic, 10 June 2015.

33. Jan Kleine Deters, Rasa Zalakeviciute, Mario Gonzalez, and Yves Rybarczyk. Modeling PM2.5 Urban Pollution Using Machine Learning and Selected Meteorological Parameters[J]. Journal of
Electrical and Computer Engineering, Volume 2017, Article ID 5106045, 14 pages.

34. Julien Brajard, Alberto Carrassi, Marc Bocquet, Laurent Bertino. Combining data assimilation and machine learning to emulate a dynamical model from sparse and noisy observations: a case study with the Lorenz 96 model[J]. Combining Data Assimilation and Machine Learning, 24 Jul 2020,1-18.

35. Tsiouri V, Kovalets I, Andronopoulos S, et al. Development and first tests of a data assimilation algorithm in a Lagrangian puff atmospheric dispersion model[J]. International Journal of Environment and Pollution, 2011, 44(1-4):147-155.

36. Tsiouri V, Kovalets I, Andronopoulos S, et al. Emission rate estimation through data assimilation of gamma dose measurements in a lagrangian atmospheric dispersion model[J].Radiation protection dosimetry, 2012, 148(1):34-44.

37. Saunier O, Mathieu A, Dither D, et al. An inverse modeling method to assess the source term of the Fukushima nuclear power plant accident using gamma dose rate observations[J].Atmospheric Chemistry and Physics, 2013, 13(22): 11403-11421.

38. Sun S D, Fang S, Liu Y, et al. Coupled Source Term Estimation Using Both Forward and Backward Method for Nuclear Power Plant Accident[C]. 2016 24th International Conference on Nuclear Engineering, 2016.

39. Sun S, Li H, Fang S. A forward-backward coupled source term estimation for nuclear power plant accident: A case study of loss of coolant accident scenario[J]. Annals of Nuclear Energy,2017, 104:6474.

40. Zhu Yanmin, Xu Ailin, sun Qiang. New progress of air quality prediction methods based on deep learning [J]. China environmental monitoring, 2020,36 (3): 10-18. 